\title{
Effects of the Balanced Gap Technique on Femoral Component Rotation in TKA
}

\author{
Petra J. C. Heesterbeek MSc, Wilco C. H. Jacobs MSc, \\ Ate B. Wymenga MD, PhD
}

Received: 31 January 2008/Accepted: 10 September 2008/Published online: 2 October 2008

(C) The Association of Bone and Joint Surgeons 2008

\begin{abstract}
Femoral component rotation from a total knee prosthesis can be determined by either a measured resection technique or a balanced gap technique. With the balanced gap implantation technique, femoral component rotation can vary freely within the restrictions produced by soft tissue structures. Because internal rotation might cause patella problems, the effect of ligament releases on femoral component rotation in a prospective clinical study was studied. Femoral component rotation was measured intraoperatively with a tensor applied in flexion at $150 \mathrm{~N}$ in 87 knees. Great interpatient variability was found; femoral component rotation, reference from the posterior condyles, ranged from $-4^{\circ}$ to $13^{\circ}$. There was no difference in femoral component rotation of knees with or without ligament releases in extension. However, knees with major medial release had less external femoral component rotation than knees with minor lateral releases. Preoperative alignment had no influence on femoral component rotation. The use of the balanced gap implantation technique theoretically will result in a balanced flexion gap, but the amount of femoral component rotation will be variable owing to patient variability and variation in ligament releases.
\end{abstract}

Each author certifies that he or she has no commercial associations (eg, consultancies, stock ownership, equity interest, patent/licensing arrangements, etc) that might pose a conflict of interest in connection with the submitted article.

P. J. C. Heesterbeek ( $₫)$, W. C. H. Jacobs

Department of Research, Development \& Education,

Sint Maartenskliniek, Postbox 9011, 6500 GM Nijmegen,

The Netherlands

e-mail: p.heesterbeek@maartenskliniek.nl

A. B. Wymenga

Department of Orthopaedics, Sint Maartenskliniek, Nijmegen,

The Netherlands
Level of Evidence: Level II, therapeutic study. See the Guidelines for Authors for a complete description of levels of evidence.

\section{Introduction}

Numerous authors have described a causal relation between malrotation of the femoral component and patellofemoral complications in TKA $[1,3,4,7,18,23]$. Malrotation of the femoral component may cause torsional stress on the tibia component that may lead to wear or loosening of the prosthesis [22]. Femoral component rotation of a total knee prosthesis can be determined by two techniques or philosophies: a measured resection technique and a balanced gap technique. Until now, there has been no consensus regarding which technique results in a better outcome.

With measured resection techniques, the rotation of the femoral component is fixed regardless of the tension of the ligaments. The three most commonly used measured resection techniques are the transepicondylar axis, the anteroposterior line or Whiteside's line, and the posterior condyles with $3^{\circ}$ external rotation as references to rotationally position the femoral component $[5,13,21,22,26]$. The flexion gap can be either rectangular or trapezoid. However, the philosophy of the classic balanced gap approach is the knee must be balanced (ie, equal tension in medial and lateral soft tissue knee structures) in extension and flexion to achieve proper kinematics and stability of the knee [6]. With the balanced gap technique, first described by Insall et al. in 1976, the soft tissue structures of the knee are tensed in flexion after ligamentous release in extension [8, 9]. In extension, the tight ligamentous structures are released until the alignment of the knee is 
neutral. In $90^{\circ}$ flexion, a tensor or a laminar spreader is inserted in the knee. The anterior and posterior femur cuts will be performed parallel to the tibia cut while the knee is distracted with a tensor. As a consequence of this balanced gap technique, the rotation of the femoral component can vary freely within the restrictions of the soft tissue structures. Flexion gap balancing is important for a stable knee in flexion [10] and better range of motion [17, 19].

Owing to the arthritic process and/or a preexisting deformity, a knee may have fixed varus or valgus alignment. If so, release of the lateral or medial soft tissue structures is necessary to create a straight leg. When a ligament structure has a stabilizing effect in extension and flexion, release of this structure in extension will also loosen the structure in flexion. Krackow and Mihalko reported ligament releases performed in extension may have a larger effect in flexion [15, 16]. After release in extension, when the flexion gap is distracted to create a rectangular flexion gap, soft tissue tension on the released side is decreased. The joint space at the released side will open more and the femur may rotate externally under tension after medial release and internally after lateral release (Fig. 1).

The consequence of the balanced gap technique is variation of femoral component rotation within the limits imposed by the soft tissue tension. Because release of
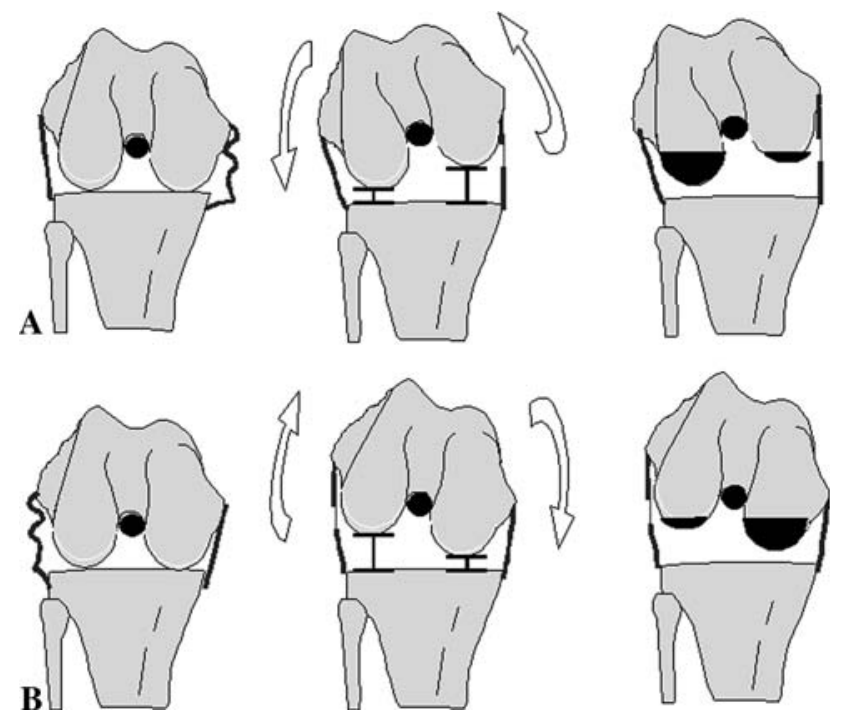

Fig. 1A-B The theoretical effect of ligament releases on femoral component rotation is shown. (A) For medial ligament release, when the tensor is inserted in the knee, the femur will exorotate as a result of the enlarged medial ligament. Consequently, the femur component of the prosthesis will be placed in endorotation with respect to the femur because the posterior cut is parallel to the tibia cut. (B) For a lateral ligament release, when the tensor is inserted in the knee, the femur will endorotate as a result of the enlarged lateral ligament. Consequently, the femur component of the prosthesis will be placed in exorotation with respect to the femur as the posterior cut is parallel to the tibia cut. these structures may be necessary to obtain neutral alignment in extension, there has been some concern that using the balanced gap technique could lead to (undesirable) internal rotation of the femoral component in some knees and could cause patellofemoral maltracking [6]. We designed this prospective clinical study to investigate whether ligament releases in extension lead to undesired femoral component rotation in flexion when the balanced gap technique is used.

The primary research question of this clinical study using the balanced gap technique in posterior cruciate ligament (PCL)-retaining TKA is, do ligament releases in extension lead to a different amount of femoral component rotation? We hypothesized that medial ligament releases will result in more internal rotation of the femoral component compared with lateral releases (Fig. 1). The second question is, does the side and extent of the ligament release have an effect on the amount of femoral component rotation? We hypothesized that the greater the extent of the release, the greater the rotating effect of the release. The third question is, does preoperative leg alignment relate to femoral component rotation? We expected more internal rotation in varus and more external rotation in valgus knees.

\section{Materials and Methods}

In this prospective clinical cohort study, 83 consecutive patients with primary osteoarthritis undergoing a primary TKA were included. Inclusion criteria were $15^{\circ}$ fixed varus or valgus alignment maximum and an intact PCL. Preoperative leg alignment was determined by a blinded researcher $(\mathrm{PH})$ on standardized full-leg standing radiographs. Patients were excluded from the study if they had an osteotomy, ligament lesions, or previous ligament surgery. During surgery, the PCL was evaluated after tibial resection. We excluded patients with a ruptured or macroscopically insufficient PCL. Eighty-seven knees (41 left knees, 46 right knees) in 83 consecutive patients (average age, $65.8 \pm 9.2$ years; 19 males, 64 females) who underwent primary TKA were evaluated. Measurements could be performed in all 87 knees. In 40 knees, no releases were necessary to align the leg other than the minor releases to expose the joint as mentioned below. Among these knees, 18 were left and 22 were right knees; there were 34 women and six men. Preoperative alignment was valgus for six knees, neutral for 18 , and varus for 16 knees. Ligament releases necessary to align the leg in extension were performed in 47 knees. These were 23 left knees and 24 right knees in 34 female and 13 male patients. Before surgery, 12 knees were in valgus, nine in neutral, and 26 in varus alignment. 
We implanted a total condylar cruciate-retaining knee prosthesis (balanSys $^{\mathrm{TM}}$; Mathys Ltd, Bettlach, Switzerland) using the balanSys ${ }^{\mathrm{TM}}$ soft tissue tensor applied in flexion and extension. Surgery was performed by one surgeon (AW) according to the balanced gap technique surgical protocol. A medial approach was used in varus knees and a lateral approach in valgus knees. For the knees with neutral alignment (ie, alignment close to $180^{\circ}$ hip-knee-ankle angle), the surgeon chose either the medial or the lateral approach. With the medial approach, the deep medial collateral ligament was released after medial arthrotomy by removing approximately $1 \mathrm{~cm}$ of periosteum from the tibia to remove the osteophytes. In lateral arthrotomies, $1 \mathrm{~cm}$ periosteum from the tibia and the tractus iliotibialis was released. All osteophytes were removed. If present, the anterior cruciate ligament was excised. An external tibial alignment system attached to the proximal tibia and the ankle and aligned to the second metatarsal was used in every patient. A perpendicular tibia cut was performed according to the standard operative protocol. Thereafter, the balanSys tensor ${ }^{\mathrm{TM}}$ (Mathys Ltd) was inserted into the knee to balance the extension gap at $200 \mathrm{~N}$ to achieve neutral leg alignment as determined by the surgeon and the intramedullary femur guide at $5^{\circ} / 6^{\circ} / 7^{\circ}$ based on hip-kneeankle radiographs. With this bicompartmental tensor, the medial and lateral compartment of the knee could be distracted separately with a measured amount of force. If necessary to align the knee, ligament releases were performed in extension. Releases were not performed following a fixed sequence; the surgeon consistently chose to release the tightest ligament first, because this ligament was the first inhibitor to a straight leg. After the first ligament release, the knee was again tensioned in extension. If the knee still was malaligned, a second release was performed; again, the tightest structure at that time was released. All releases performed were registered and catalogued to be used as independent variables (Table 1). Inherent to this balanced gap technique is that after soft tissue balancing in extension, no additional releases will be performed in flexion. The tensor was inserted into the knee in $90^{\circ}$ flexion and $150 \mathrm{~N}$ was applied; the same tension applied to the medial and lateral soft tissue structures determined the flexion gap. The amounts of force of $200 \mathrm{~N}$ in extension and $150 \mathrm{~N}$ in flexion to tension the knee were chosen based on the surgeon's experience and were consistently applied.

To determine femoral component rotation, a cutting jig with $3^{\circ}$ external rotation was used to simulate a $3^{\circ}$ external rotation bone cut referenced from the posterior condyles (Fig. 2). A line was drawn on the uncut articular distal surface of the femur using electrocautery (Fig. 2A). After that, the tensor was inserted in $90^{\circ}$ flexion and the flexion gap was distracted with $150 \mathrm{~N}$. The femur bone rotated freely based on the soft tissue tension (Fig. 2C). A custommade goniometer was attached to the external tibial alignment system (Fig. 2B). Zero degrees on the goniometer represented the femoral component rotation (parallel to the tibia cut) as determined with the balanced gap technique. In this position with the tensor in place, the angle of the previously marked $3^{\circ}$ line was measured with the goniometer (Fig. 2D); this is the difference in femoral component rotation between the $3^{\circ}$ external rotation cut referenced from the posterior condyles and the amount of rotation using the balanced gap technique. Later, the number of degrees of the balanced gap line referenced from the posterior condyles (eg, a line parallel to the posterior condylar line is $0^{\circ}$ ) could be calculated by adding the $3^{\circ}$ external rotation. The amount of femoral rotation was the dependent variable in the analysis. All measurements could be performed to the nearest $1^{\circ}$. In our opinion, rotation referenced from the posterior condyles could be assessed accurately during surgery and did not introduce observerbased inaccuracy. External rotation was defined as a positive value and internal rotation as a negative value.

To investigate the influence of ligament releases on femoral component rotation, after the surgeries, we divided knees into two categories: knees without ligament release and knees with any ligament release. For this categorization, releases were defined as procedures performed to influence the alignment of the leg in extension. Four

Table 1. Classification release categories*

\begin{tabular}{llll}
\hline Side & Classification & Structure(s) & Technique \\
\hline Medial & Minor & Posteromedial capsule & Release from tibia \\
& Major & Superficial medial collateral ligament & Release from tibia \\
& Major & Semimembranosus & Release from tibia \\
Lateral & Minor & Lateral posterior capsule & Release from tibia \\
& Major & Popliteus tendon & Release from femur insertion \\
& Major & Fibular ligament & Release from femur insertion \\
& Major & Lateral intermuscular septum on femur & Release from femur insertion \\
\hline
\end{tabular}

*A knee was classified as needing a major release if one or more of the performed releases were in the major category. 




Fig. 2A-D A stepwise illustration for measurement of femoral component rotation is shown. (A) A line parallel to $3^{\circ}$ exorotation referenced from the posterior condyles was marked on the distal femur using a rigid block and electrocautery. (B) A custom-made goniometer was rigidly attached to the external tibia reference system. (C) The ligament tensor is inserted into the knee and $150 \mathrm{~N}$ is applied

categories of ligament releases were distinguished: medial minor release, medial major release, lateral minor, and lateral major release based on the extent of the release (Table 1). To investigate the influence of preoperative alignment, we further subclassified the knees into three groups: valgus (less than $-3^{\circ}$ mechanical axis), neutral $\left(-3^{\circ}\right.$ to $\left.3^{\circ}\right)$, and varus (greater than $\left.3^{\circ}\right)$. The number of knees that had a femoral component rotation less than $3^{\circ}$ and those less than $0^{\circ}$ (exo)rotation were determined to make a distinction between somewhat (less than $3^{\circ}$ exorotation) and more (less than $0^{\circ}$ ) internally rotated femur components. The frequency that each structure was released in the study cohort was determined in the category knees with ligament releases.

We analyzed differences in femoral component rotation between the group of knees that did not have ligament releases and the group that had ligament releases for statistical significance using the Student's t-test. To analyze the relation between the four categories of releases and to the medial and lateral compartment. (D) Rotation resulting in a rectangular flexion gap (ie, rotation parallel to the tibia cut) is $0^{\circ}$. The handle of the goniometer can be placed parallel to the marked posterior condylar line. By knowing the relative rotation between the two lines, the rotation of the balanced gap line can be calculated referenced from the posterior condyles.

femoral component rotation, and between preoperative alignment and femoral component rotation, one-way ANOVA was used. Differences in femoral component rotation between knees that had a medial or a lateral approach were tested with a Student's t-test. Results are presented as mean ( \pm standard deviation) with an $\alpha$ level of 0.05 considered significant.

\section{Results}

Using the balanced gap technique, the mean femoral component rotation for knees with ligament releases did not differ from that for knees that did not need releases. Mean femoral component rotation was $5^{\circ}\left( \pm 3.2^{\circ}\right)$ ranging from $-3^{\circ}$ to $12^{\circ}$ for knees that did not need ligament releases and $4^{\circ}\left( \pm 4.3^{\circ}\right)$ ranging from $-4^{\circ}$ to $13^{\circ}$ for knees that needed ligament releases (Fig. 3). There was high interpatient variability in femoral component rotation 
(Fig. 3). Of all knees in the no-release group, 10 (25\%) had femoral component rotation less than $3^{\circ}$, whereas one had rotation less than $0^{\circ}$. In the release group, approximately half of the knees ( 23 knees [49\%]) had femoral component rotation less than $3^{\circ}$. Of these 23 knees, eight knees had rotation less than $0^{\circ}$.

However, the type of release influenced femoral component rotation; knees with major medial releases showed the least mean external rotation $\left(2^{\circ} \pm 4.2^{\circ}\right)$ compared with knees with minor lateral releases that had the most mean external rotation $\left(7^{\circ} \pm 3.8^{\circ} ; \mathrm{p}=0.050\right)$ (Table 2$)$.

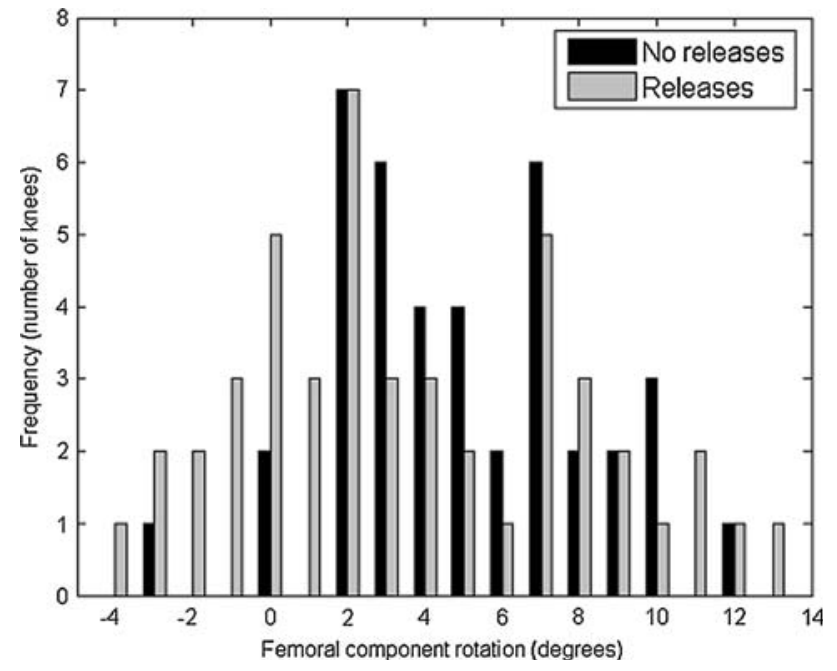

Fig. 3 In this histogram, the frequency of femoral component rotation using the ligament-guided technique with a tensor is shown for knees with no release $(n=40)$ and knees with releases $(n=47)$. Femoral component rotation is referenced from the posterior condyles. Positive rotation is external rotation; negative rotation is internal rotation.
Preoperative alignment had no influence on femoral component rotation for both knees either with or without releases (Table 3). For knees without release, mean femoral component rotation was not significantly different for medial and lateral approaches: $5^{\circ}\left( \pm 2.9^{\circ}\right)$ for a medial approach and $7^{\circ}\left( \pm 4.4^{\circ}\right)$ for a lateral approach. For knees with a release, there was perfect correlation between the approach and side of releases; in all knees with medial releases, the joint was approached medially and in all knees with lateral releases laterally. Mean femoral component rotation was significantly less for knees with a medial approach and medial release $\left(3^{\circ} \pm 4.2^{\circ}\right)$ compared with rotation in knees with a lateral approach and lateral release $\left(6^{\circ} \pm 4.2^{\circ} ; \mathrm{p}=0.039\right)$.

The frequency of releases needed in the 47 knees of the release group were one (31 knees), two (11 knees), three (three knees), and four (four knees). For medial releases in varus knees, release of the medial superficial ligament was the most frequent together with release of the posteromedial capsule. For lateral releases in valgus knees, release of the lateral posterior capsule was the most frequent (Table 4).

\section{Discussion}

In this study, we investigated whether ligament releases to obtain neutral alignment in extension using the balanced gap technique influenced femoral component rotation. A high interpatient variability in femoral component rotation was found regardless whether ligament releases were necessary. Therefore, the high variability cannot be explained by ligament releases alone. We suspect the status of the ligaments and the laxity before the operation were

Table 2. Mean femoral component rotation $( \pm \mathrm{SD})$ for medial and lateral release categories*

\begin{tabular}{llll}
\hline Release category & $\begin{array}{l}\text { Mean femoral component } \\
\text { rotation }( \pm \mathrm{SD})\end{array}$ & Category & $\begin{array}{l}\text { Mean femoral component } \\
\text { rotation }( \pm \mathrm{SD})\end{array}$ \\
\hline Medial, overall $(\mathrm{n}=32)$ & $3^{\circ}\left( \pm 4.2^{\circ}\right)^{\dagger}$ & Minor $(\mathrm{n}=5)$ & $5^{\circ}\left( \pm 4.0^{\circ}\right)$ \\
& & Major $(\mathrm{n}=27)$ & $2^{\circ}\left( \pm 4.2^{\circ}\right)^{\ddagger}$ \\
Lateral, overall $(\mathrm{n}=15)$ & $6^{\circ}\left( \pm 4.2^{\circ}\right)$ & Minor $(\mathrm{n}=10)$ & $7^{\circ}\left( \pm 3.8^{\circ}\right)$ \\
& & Major $(\mathrm{n}=5)$ & $4^{\circ}\left( \pm 4.6^{\circ}\right)$ \\
\hline
\end{tabular}

*Femoral component rotation was referenced from the posterior condyles and positive rotation is external rotation; ${ }^{\dagger}$ medial versus lateral releases $\mathrm{p}$ value $=0.039 ;{ }^{*}$ overall $\mathrm{p}$ value $=0.050$, post hoc difference between medial major and lateral minor, $\mathrm{p}$ value $=0.050 ; \mathrm{SD}=\mathrm{standard}$ deviation.

Table 3. Mean femoral component rotation $( \pm \mathrm{SD})$ for preoperative alignment*

\begin{tabular}{lllll}
\hline Category & Overall & Valgus & Neutral & Varus \\
\hline No releases $(\mathrm{n}=40)$ & $5^{\circ}\left( \pm 3.2^{\circ}\right)$ & $6^{\circ}\left( \pm 4.1^{\circ}\right)$ & $5^{\circ}\left( \pm 3.3^{\circ}\right)$ & $4^{\circ}\left( \pm 2.8^{\circ}\right)$ \\
Releases $(\mathrm{n}=47)$ & $4^{\circ}\left( \pm 4.3^{\circ}\right)$ & $6^{\circ}\left( \pm 3.6^{\circ}\right)$ & $3^{\circ}\left( \pm 4.3^{\circ}\right)$ & $3^{\circ}\left( \pm 4.5^{\circ}\right)$ \\
\hline
\end{tabular}

*Femoral component rotation was referenced from the posterior condyles and positive rotation is external rotation; SD = standard deviation. 
Table 4. Frequencies of medial and lateral releases*

\begin{tabular}{lc}
\hline Ligament & Frequency \\
\hline Posteromedial capsule & 18 \\
Medial superficial ligament & 24 \\
Medial semimembranosus & 6 \\
Lateral posterior capsule & 13 \\
Lateral popliteal tendon & 3 \\
Lateral fibular ligament & 3 \\
Lateral intermuscular septum on femur & 3 \\
\hline
\end{tabular}

*The deep medial ligament is always released during arthrotomy in varus knees and the lateral tractus iliotibialis is always released during arthrotomy in valgus knees and therefore are omitted.

highly variable and were the main factors causing the variation in femoral component rotation when the balanced gap approach was used. Consequently, the type and number of releases performed alone will not predict the final rotational position of the femoral component for this balanced gap technique. Our hypothesis that a medial release would externally rotate the femur under distracting force of the tensor and consequently this would lead to internal rotation of the femoral component when a rectangular flexion gap is created could be confirmed; medial releases led on average to less external rotation than lateral releases. Furthermore, major medial releases led to the least external rotation, again confirming our hypothesis. However, on the lateral side, minor lateral releases led to more external femoral component rotation than major lateral releases. This was not expected because major lateral releases, with more ligament removed, theoretically would lead to the most external femoral component rotation (Fig. 1B). A possible explanation may be the low frequency of major lateral releases in this study $(n=5)$. Another explanation is that even in knees with complete release of the femoral insertion of the fibular ligament and the popliteal tendon, some tissue connections such as the fabellofibular ligament still remain on the dorsal lateral side between the femur and tibia, thus preventing complete lateral instability in flexion. Furthermore, the medially stabilizing property of the intact PCL may help prevent excessive internal rotation of the femur followed by external rotation of the femoral component. Another unexpected result was preoperative alignment as measured on a hip-knee-ankle radiograph had no influence on rotation. This was unexpected because axial malalignment theoretically would need release on the concave side with effects on rotation.

The approach, medial or lateral, had a major influence on femoral component rotation in the knees with ligament releases. However, because there was a one-to-one relationship between approach and side of the release, this might be an artificial effect with the ligament releases actually causing the effect on femoral component rotation.
In addition, there is no anatomic explanation for a supposed relationship between anterior structures cut during the approach of the joint and femoral component rotation that are guided mainly by collateral structures in flexion.

The fact that we used the posterior condyles as a reference might have introduced some bias. However, we do not know the effect of hypoplasia or worn posterior condyles on adapted lateral ligaments with this balanced gap technique, and thus, on the measured femoral component rotation. Apparently, it is the release that primarily affects femoral component rotation. Although bone-reference methods such as the $3^{\circ}$ external rotation or use of the transepicondylar axis may be more familiar to many orthopaedic surgeons, $3^{\circ}$ external rotation also is based on the posterior condyles, whereas the transepicondylar axis is difficult to determine intraoperatively and must be used with caution because of low reproducibility and reliability resulting from the high perioperative variance [11-13].

Another source of bias may be the demarcation and measurement of femoral component rotation. Although we did not determine the accuracy of the bone cut, we assume because the calibrated goniometer was rigidly attached to the tibial cutting block, the accuracy of the goniometer's measurements would be similar to those of the bone cut. Because rotation of the femoral component is determined by the anterior and posterior femoral cuts and these are parallel to the tibial cut, it has the following influence. If the tibial cut would be in varus, then the femoral cuts would be endorotated, which would lead to more internal rotation of the femoral component. However, the tibial cut was aligned using an external tibial reference frame and we did not detect any systematic errors, therefore, we are confident the tibial cuts were perpendicular within a few degrees as with any total knee system.

The amount of force on the tensor may have affected the amount of femoral component rotation. In this study, the amount of force used was $150 \mathrm{~N}$ in flexion. It is not clear whether this amount of force is the optimal amount. However, to our knowledge, this is the first in vivo study reporting intraoperative rotation measurements using a tensor with consistent measurable tension $(150 \mathrm{~N}$ in flexion and $200 \mathrm{~N}$ in extension) in all knees.

Femoral component rotation when using the balanced gap technique has been investigated before [6, 21, 27]; a comparable amount of femoral rotation was needed to make a symmetric flexion space using a balancing device, $4.83^{\circ} \pm 3.29^{\circ}$, also referenced to the posterior condyles [27]. There was wide variation of intraoperative femoral component rotation ranging from $-7^{\circ}$ to $8^{\circ}$ in posteriorstabilized TKAs using laminar spreaders in $90^{\circ}$ knee flexion [6]. Compared with our findings ranging from $-3^{\circ}$ to $12^{\circ}$, our results show a slight shift toward external rotation. This may be attributable to the intact PCL in our 
series, which theoretically stabilizes the femur on the medial side owing to its oblique anatomic orientation. Our in vivo finding that medial releases led to less external rotation compared with lateral releases confirms previous in vitro studies $[15,20]$.

In contrast to another study [27], we could not confirm our hypothesis that preoperative alignment influences femoral component rotation (valgus knees show more external femoral component rotation than varus knees). To some extent, our unexpected findings can be explained by the fact that a number of the deformities were acquired and not congenital and thus correctable without ligament releases in extension. Some of the deformities might have been corrected after removal of osteophytes. Some patients with a large preoperative deformity seen on standing radiographs will not need releases, whereas others with smaller axial deviations may need an extensive release to correct the leg axis.

The picture emerging from these observations is patients have large variability in ligamentous stability leading to large variability in femoral component rotation when the balanced gap technique is used, which is influenced to some extent by releases necessary to align the leg in extension. From our data, it is clear the assumption that the flexion gap is simply balanced by $3^{\circ}$ external rotation of the femoral component (or using the transepicondylar axis) is not always correct and will lead to trapezoid flexion gaps with resulting flexion instabilities [25]. This has important clinical implications because it has been shown that a well-balanced knee is a prerequisite for satisfactory postoperative proprioception [2], proper kinematics [6], and for good postoperative range of motion [19]. In case of a mobile insert, stability of the flexion and extension gap is even more important because subluxation or dislocation of the tibial liner may cause increased wear and pain [24].

When using a balanced gap technique, the knee theoretically will be balanced, but some patients will have an internally rotated femoral component at the expense of a balanced flexion gap. Although some studies investigating this subject reported a negative effect of internal component rotation on patellar tracking [1, 4, 23], there is no conclusive evidence that patellofemoral complaints are caused by a malrotated femoral component [3, 14]. It is still unknown how much internal rotation of the femoral component with a balanced gap technique could be tolerated without causing patellofemoral problems and therefore more research is needed. If one is concerned that internal rotation of the femoral component of balanced knees might create problems, one could attempt to prevent internal rotation of the femoral component beyond $0^{\circ}$ and accept that some medial flexion laxity will be present in some patients.
Our study showed when using a balanced gap implantation technique, knees with ligaments released had the same amount of femoral component rotation as knees without ligament releases. However, if releases had been performed, major medial releases led to the least and minor lateral releases to the greatest amount of femoral rotation. Preoperative alignment had no influence on femoral component rotation.

Acknowledgments We thank Hans Radenborg for taking photographs of the measurement device and Patsy Anderson for editorial comments.

\section{References}

1. Anouchi YS, Whiteside LA, Kaiser AD, Milliano MT. The effects of axial rotational alignment of the femoral component on knee stability and patellar tracking in total knee arthroplasty demonstrated on autopsy specimens. Clin Orthop Relat Res. 1993;287:170-177.

2. Attfield SF, Wilton TJ, Pratt DJ, Sambatakakis A. Soft-tissue balance and recovery of proprioception after total knee replacement. J Bone Joint Surg Br. 1996;78:540-545.

3. Barrack RL, Schrader T, Bertot AJ, Wolfe MW, Myers L. Component rotation and anterior knee pain after total knee arthroplasty. Clin Orthop Relat Res. 2001;392:46-55.

4. Berger RA, Crossett LS, Jacobs JJ, Rubash HE. Malrotation causing patellofemoral complications after total knee arthroplasty. Clin Orthop Relat Res. 1998;356:144-153.

5. Berger RA, Rubash HE, Seel MJ, Thompson WH, Crossett LS. Determining the rotational alignment of the femoral component in total knee arthroplasty using the epicondylar axis. Clin Orthop Relat Res. 1993;286:40-47.

6. Fehring TK. Rotational malalignment of the femoral component in total knee arthroplasty. Clin Orthop Relat Res. 2000;380:72-79.

7. Hofmann S, Romero J, Roth-Schiffl E, Albrecht T. [Rotational malalignment of the components may cause chronic pain or early failure in total knee arthroplasty] [in German]. Orthopade. 2003; 32:469-476.

8. Insall J, Ranawat CS, Scott WN, Walker P. Total condylar knee replacement: preliminary report. Clin Orthop Relat Res. 1976;120:149-154.

9. Insall JN, Binazzi R, Soudry M, Mestriner LA. Total knee arthroplasty. Clin Orthop Relat Res. 1985;192:13-22.

10. Insall JN, Scott WN, eds. Surgery of the Knee. Vol 2. Philadelphia, PA: Churchill Livingstone; 2001:1089.

11. Jenny JY, Boeri C. Low reproducibility of the intra-operative measurement of the transepicondylar axis during total knee replacement. Acta Orthop Scand. 2004;75:74-77.

12. Jerosch J, Peuker E, Philipps B, Filler T. Interindividual reproducibility in perioperative rotational alignment of femoral components in knee prosthetic surgery using the transepicondylar axis. Knee Surg Sports Traumatol Arthrosc. 2002;10:194-197.

13. Katz MA, Beck TD, Silber JS, Seldes RM, Lotke PA. Determining femoral rotational alignment in total knee arthroplasty: reliability of techniques. $J$ Arthroplasty. 2001;16:301-305.

14. Kawano T, Miura H, Nagamine R, Urabe K, Matsuda S, Mawatari $\mathrm{T}$, Moro-Oka T, Iwamoto Y. Factors affecting patellar tracking after total knee arthroplasty. J Arthroplasty. 2002;17:942-947.

15. Krackow KA, Mihalko WM. Flexion-extension joint gap changes after lateral structure release for valgus deformity correction in total knee arthroplasty: a cadaveric study. J Arthroplasty. 1999; 14:994-1004. 
16. Krackow KA, Mihalko WM. The effect of medial release on flexion and extension gaps in cadaveric knees: implications for soft-tissue balancing in total knee arthroplasty. Am J Knee Surg. 1999;12:222-228.

17. Laskin RS. Flexion space configuration in total knee arthroplasty. J Arthroplasty. 1995;10:657-660.

18. Matsuda S, Miura H, Nagamine R, Urabe K, Hirata G, Iwamoto Y. Effect of femoral and tibial component position on patellar tracking following total knee arthroplasty: 10-year follow-up of Miller-Galante I knees. Am J Knee Surg. 2001;14:152-156.

19. Matsuda Y, Ishii Y, Noguchi H, Ishii R. Varus-valgus balance and range of movement after total knee arthroplasty. J Bone Joint Surg Br. 2005;87:804-808.

20. Mihalko WM, Miller C, Krackow KA. Total knee arthroplasty ligament balancing and gap kinematics with posterior cruciate ligament retention and sacrifice. Am J Orthop. 2000;29:610-616.

21. Olcott CW, Scott RD. A comparison of 4 intraoperative methods to determine femoral component rotation during total knee arthroplasty. J Arthroplasty. 2000;15:22-26.
22. Poilvache PL, Insall JN, Scuderi GR, Font-Rodriguez DE. Rotational landmarks and sizing of the distal femur in total knee arthroplasty. Clin Orthop Relat Res. 1996;331:35-46.

23. Rhoads DD, Noble PC, Reuben JD, Mahoney OM, Tullos HS. The effect of femoral component position on patellar tracking after total knee arthroplasty. Clin Orthop Relat Res. 1990;260:43-51.

24. Ridgeway S, Moskal JT. Early instability with mobile-bearing total knee arthroplasty: a series of 25 cases. J Arthroplasty. 2004;19:686-693.

25. Romero J, Duronio JF, Sohrabi A, Alexander N, MacWilliams BA, Jones LC, Hungerford DS. Varus and valgus flexion laxity of total knee alignment methods in loaded cadaveric knees. Clin Orthop Relat Res. 2002;394:243-253.

26. Whiteside LA, Arima J. The anteroposterior axis for femoral rotational alignment in valgus total knee arthroplasty. Clin Orthop Relat Res. 1995;321:168-172.

27. Winemaker MJ. Perfect balance in total knee arthroplasty: the elusive compromise. J Arthroplasty. 2002;17:2-10. 